\title{
New functionalities of Maillard reaction products as emulsifiers and encapsulating agents, and the processing parameters: a brief review
}

\begin{abstract}
Non-enzymatic browning has been a wide and interesting research area in the food industry, ranging from the complexity of the reaction to its applications in the food industry as well as its ever-debatable health effects. This review provides a new perspective to the Maillard reaction apart from its ubiquitous function in enhancing food flavour, taste and appearance. It focuses on the recent application of Maillard reaction products as an inexpensive and excellent source of emulsifiers as well as superior encapsulating matrices for the entrapment of bioactive compounds. Additionally, it will also discuss the latest approaches employed to perform the Maillard reaction as well as several important reaction parameters that need to be taken into consideration when conducting the Maillard reaction.
\end{abstract}

Keyword: Maillard reaction; Maillard reaction products (MRPs); Application; Microencapsulation emulsion 